\title{
Electrical conductivity and Young's modulus of flexible nanocomposites made by metal-ion implantation of polydimethylsiloxane: The relationship between nanostructure and macroscopic properties
}

\author{
M. Niklaus, H.R. Shea* \\ Ecole Polytechnique Fédérale de Lausanne (EPFL), Microsystems for Space Technologies Laboratory (LMTS), rue Jaquet Droz 1,CP 526, \\ CH-2002 Neuchâtel, Switzerland
}

Received 22 September 2010; received in revised form 11 October 2010; accepted 12 October 2010

Available online 4 November 2010

\begin{abstract}
The mechanical and electrical properties of nanocomposites created by gold and titanium implantation into polydimethysiloxane (PDMS) are reported for doses from $10^{15}$ to $5 \times 10^{16}$ at. $\mathrm{cm}^{-2}$, and for ion energies of $2.5,5$ and $10 \mathrm{keV}$. Transmission electron microscopy (TEM) cross-section micrographs allowed detailed microstructural analysis of the implanted layers. Gold ions penetrate up to $30 \mathrm{~nm}$ and form crystalline nanoparticles whose size increases with ion dose and energy. Titanium forms a nearly homogeneous amorphous composite with the PDMS up to $18 \mathrm{~nm}$ thick. Using TEM micrographs, the metal volume fraction of the composite was accurately determined, allowing both electrical conductivity and Young's modulus to be plotted vs. the volume fraction, enabling quantitative use of percolation theory for nanocomposites $<30 \mathrm{~nm}$ thick. This allows the composite's Young's modulus and conductivity to be linked directly to the implantation parameters and volume fraction. Electrical and mechanical properties were measured on the same nanocomposite samples, and different percolation thresholds and exponents were found, showing that, while percolation explains both conduction and stiffness of the composite very well, the interaction between metal nanoparticles occurs differently in determining mechanical and electrical properties.
\end{abstract}

(C) 2010 Acta Materialia Inc. Published by Elsevier Ltd. All rights reserved.

Keywords: Polymer matrix composites; Nanostructured materials; Elastic constant; Electrical conductivity; Electro-active polymers

\section{Introduction}

Composites can combine the properties of its constituents in a very advantageous manner. Polymer-metal composites, generally made by mixing conductive particles in the uncured elastomer [1-4] are of interest in the rapidly growing field of flexible and stretchable electronics. Another technique to make polymer-metal composites is metal-ion implantation of polymers, which leads to the formation of metal nanoparticles in the top $10-100 \mathrm{~nm}$ of the

\footnotetext{
* Corresponding author. Tel.: +41 327205584.

E-mail address: herbert.shea@epfl.ch (H.R. Shea).
}

polymer, creating a metal/insulator composite whose electrical conductivity displays percolative behavior $[5,6]$.

The authors' group showed previously that $5 \mathrm{keV}$ implantation of gold ions into the elastomer polydimethlysiloxane (PDMS) is an effective method of fabricating compliant stretchable electrodes, patternable on the micrometer scale, conducting at uniaxial strains $>175 \%$ and remain operational after $>10^{5}$ cycles at $30 \%$ strain [7]. These gold-PDMS composite electrodes were then used to fabricate miniaturized dielectric electroactive polymer actuator with very large displacement [8].

The ion implantation leads to the creation of metal nanoclusters (1-20 nm diameter) embedded in the PDMS (see the TEM mage in Fig. 1). By operating just above 


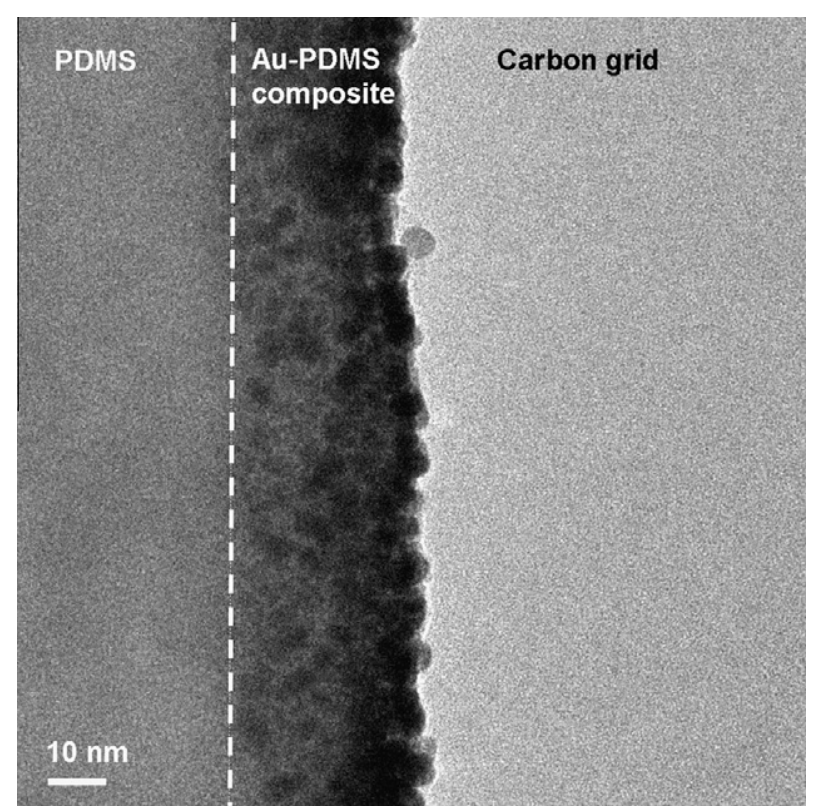

Fig. 1. TEM cross-section micrograph of PDMS implanted with gold ions at $10 \mathrm{keV}$ and a dose of $1.5 \times 10^{16}$ at. $\mathrm{cm}^{-2}$. The implantation direction was from right to left. The composite is visible just below the surface of the PDMS as the region with dark clusters. The white dashed line indicates the boundary between implanted (composite) and non-implanted layer used in this paper for calculation of the volume fraction.

the percolation threshold, one can combine good conductivity with minimal stiffening of the elastomer. The metal clusters touch and provide an electronic conductive path, without forming a strong mechanical bond. This absence of a strong bond between clusters allows them to slide relative to each other if strain is applied to the polymer matrix [8], allowing for important strains before losing conduction and low degradation when cyclically stretched.

As these nanometer-thick metal-elastomer composites are unique in the field of the conductor-insulator materials from the point of view of fabrication, and also highly anisotropic, the commonly used theory describing the physical properties of such systems is only of limited help in understanding and describing the measured characteristics $[9,10]$. The system is of particular interest because both electrical and mechanical properties are measured on the same samples, and thus percolation theory is applied here to two different properties, giving an important indication of how percolation theory applies differently to the same system for the mechanical and electrical properties.

This work measures and models the electrical conductivity $\sigma$ and Young's modulus $Y$ of a polymer-metal composite, created by metal-ion implantation of PDMS, and relates $\sigma$ and $Y$ to the composite's nanostructure, which is obtained from TEM cross-sections. The metals are gold and titanium, implanted at $2.5,5$ and $10 \mathrm{keV}$, with doses ranging between $0.1 \times 10^{16}$ and $5 \times 10^{16}$ at. $\mathrm{cm}^{-2}$. Since the discussion of the physical properties will be related to the structure results, the TEM analysis of the composites is first presented in Section 4.1. This TEM study is essential for the model, as it allows an accurate determination of the metal volume fraction. In Sections 4.2 and 4.3, the data for $\sigma$ and the $Y$ are discussed, concluding with a comparison of the percolation model for the two physical properties.

\section{Theory}

The literature on the mechanical and electrical properties of heterogeneous materials is vast [11]. Many models have been proposed for the electrical conductivity $\sigma$ and Young's modulus $Y$ of heterogeneous material, using analytical or numerical approaches for many possible configurations (concentration, material, polydispersivity, fabrication method, etc.) [12]. In this work, the laminar and percolation theories are used [13], which are derived for homogeneous particle distribution, i.e. for isotropic materials and composites. Salvadori et al. modelled the conductivity of the gold-implanted PMMA with percolation theory [5].

\subsection{Percolation theory and general effective media}

Percolation theory is based on a system in which there is a volume fraction $\phi$ of particles in a matrix. Increasing the concentration of particles leads to the formation of chains which, at the percolation threshold (transition point), form chains spanning the entire system. The theory splits into discrete and continuous systems. For conductivity and Young's modulus, the governing equations have the form:

$$
\begin{array}{lr}
\sigma_{\mathrm{Comp}}=\sigma_{c}\left(\phi-\phi_{c}\right)^{t} \text { and } Y_{\mathrm{Comp}}=Y_{c}\left(\phi-\phi_{c}\right)^{f} & \text { for }\left[\phi>\phi_{t}\right] \\
\sigma_{\mathrm{Comp}}=\sigma_{i}\left(\phi_{c}-\phi\right)^{s} \text { and } Y_{\mathrm{Comp}}=Y_{i}\left(\phi_{c}-\phi\right)^{a} & \text { for }\left[\phi<\phi_{c}\right]
\end{array}
$$

where $\sigma, Y$ and $\phi$ are the electrical conductivity, Young's modulus and the volume fraction of the particles, respectively. Index c stands for conductor, $i$ for insulator, and Comp for the conductor-insulator composite. $\phi_{c}$ is the critical volume fraction, i.e. the percolation threshold. Numerical simulations and experiments for two-dimensional (2D) yield: $t=s \approx 1.3, f \approx 3.96, a \approx 1.24$; and for three-dimensional $(3 \mathrm{D}): t \approx 2, s \approx 0.87, f \approx 3.75, a \approx 0.65[14,15]$. It has been claimed that $\phi_{c}$ and the exponents from the lattice (simulation) and real continuum media belong to the same universal class and depend only on the dimensionality of the system [16,17]. However, it has been shown that the universal values for continuum systems do not exist, as they depend on the details of the microstructure and the model used, and not only on the dimension of the system $[18,19]$.

McLachlan et al. proposed the general effective media (GEM) equation, which describes the percolation mechanism of a binary composite system over the whole scale of the volume fraction [20]: 
$\frac{(1-\phi)\left(Y_{i}^{1 / a}-Y_{\text {Comp }}^{f / a}\right)}{Y_{i}^{1 / a}-A Y_{\text {Comp }}^{1 / a}}+\frac{\phi\left(Y_{c}^{1 / f}-Y_{\text {Comp }}^{1 / f}\right)}{Y_{c}^{1 / f}+A Y_{\text {Comp }}^{1 / f}}=0$

with $A=\left(1-\phi_{c}\right) / \phi_{c}$ for $0 \leqslant \phi_{c} \leqslant 1$.

GEM is based on the percolation and effective medium theories, and one can see that Eq. (2) reduces to the Eqs. (1a) and (1b) when $Y_{c}\left(\sigma_{c}\right) \rightarrow \infty$ or $Y_{i}\left(\sigma_{i}\right)=0$.

\subsection{Laminar theory}

The laminar theory is only applicable for stacks of different materials [21]. If a composite is a stack of two components' layers with their heights $h_{1}$ and $h_{2}$ such that $h_{1}+h_{2}=1$, weighted arithmetic-average estimates the effective property (e.g. Young's modulus $Y$ ) of the composite by

$Y_{1} h_{1}+Y_{2} h_{2}=Y$

This equation will allow $Y$ of the implanted layer to be separated from the non-implanted layer.

\section{Experimental setup and procedure}

\subsection{Fabrication of the samples: implantation}

The implantations of the samples were performed at pressures $<8 \times 10^{-6}$ mbar with a filtered cathode vacuum arc (FCVA) plasma source, based on an RHK Technology ARC 20 pulsed arc source [22]. The ion implanter used is described in Ref. [8] and is summarized below, and differs primarily from the standard FCVA plasma gun, which emits ions with energies of order $50 \mathrm{eV}$ by the addition of a biased sample holder which provides several kilovolts energy to the ions. The system is shown schematically in Fig. 2a. Fig. 2b is a photograph taken during one pulse.
The cathode (see Fig. 2a) is the source of the ions, and consists of a 3-mm-diameter metal cylinder, held at $-600 \mathrm{~V}$ relative to the anode using a storage capacitor. The plasma is initiated by a very short $10-18 \mathrm{kV}$ voltage pulse on the trigger electrode, which allows a stable plasma to be established between the anode and the cathode during the $600 \mu$ s that it takes for the storage capacitor to discharge at a current of up to $100 \mathrm{~A}$. The FCVA is thus operated in a pulsed mode; a $2 \mathrm{~Hz}$ pulse rate was used. The plasma drifts past the anode into an electromagnetic filter, through which only ions exit the $90^{\circ}$ bend. Ion energy at the exit of the filter is of order $50 \mathrm{eV}$ [23]. The sample to be implanted is placed on a metal sample holder $2-5 \mathrm{~cm}$ below the exit of the filter (see Fig. 2b), which can be biased up to $-5 \mathrm{kV}$ relative to the anode, providing an accelerating potential for the ions. Since nearly all ions are doubly charged in this system, the maximum attainable acceleration energy is $10 \mathrm{keV}$.

The energy of the ions from such an FCVA source is not constant during each pulse. During each $600 \mu$ s pulse, the accelerating electrical field drops owing to the impact of $3 \times 10^{13}$ at. $\mathrm{cm}^{-2}$ of doubly charged gold ions on the sample being implanted. This is a common property of FCVA [24]. The first ions to reach the sample have the full $(2 \times$ bias voltage) energy, while the last ions will have a lower energy.

The substrate is a $30 \mu \mathrm{m}$ thick film of the elastomer PDMS (Sylgard 186, Dow Corning). The implanted elements are gold and titanium. The ion doses range from $0.1 \times 10^{16}$ to $5 \times 10^{16}$ at. $\mathrm{cm}^{-2}$, and three energies, 2.5, 5 and $10 \mathrm{keV}$, were applied (corresponding to accelerating biases of $1.25,2.5$ and $5 \mathrm{kV}$ ). The ion doses were determined after the implantations, using Rutherford backscattering spectrometry.

The nanocomposite is formed in approximately the top $50 \mathrm{~nm}$ of the $30 \mu \mathrm{m}$ thick PDMS film.
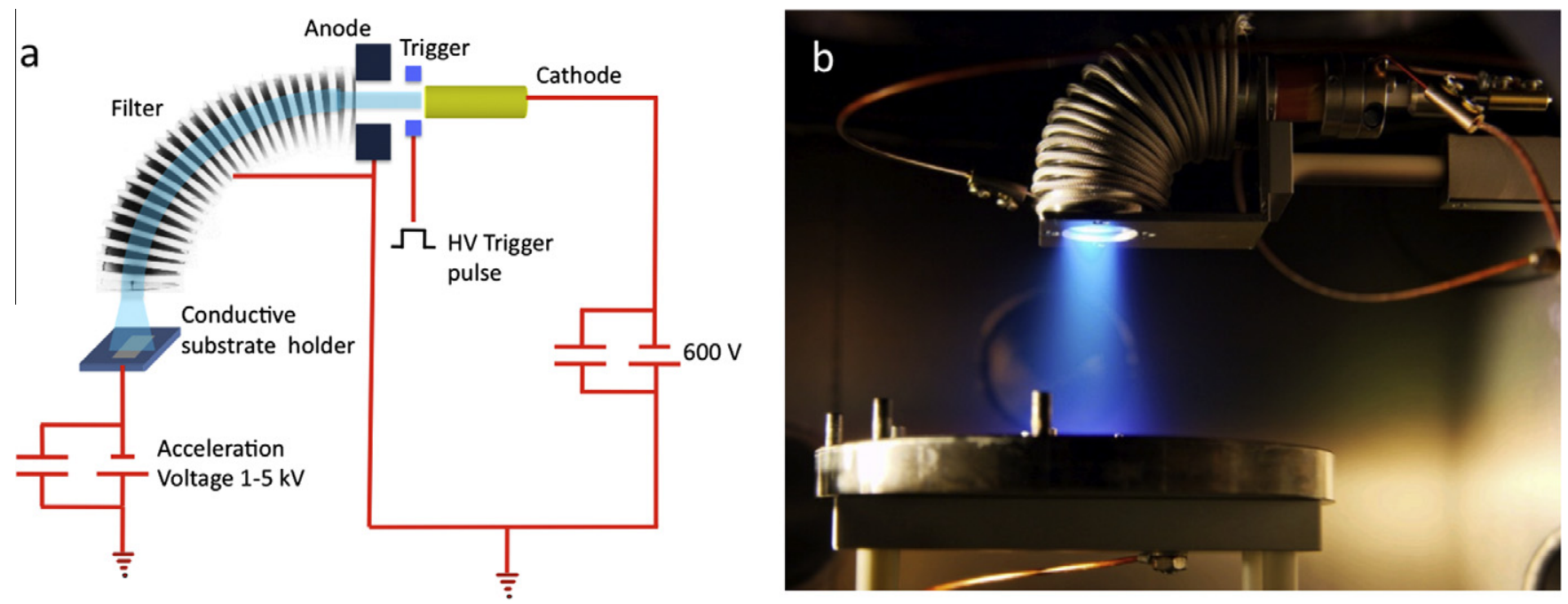

Fig. 2. Filtered cathode vacuum arc used for Ti and Au ion implantations: (a) schematic diagram of main components and applied electrical potentials; (b) photo of plasma coming out of the $90^{\circ}$ filter. 


\subsection{Electrical conductivity measurements}

The samples for the conductivity measurements consist of $30 \mu \mathrm{m}$ thick layer of PDMS bonded on a $1 \mathrm{~cm}^{2}$ Pyrex chip. The implantation is performed through a stainless steel mask, placed in contact on the top of the sample, with an opening of $0.5 \times 0.5 \mathrm{~cm}^{2}$. After the implantation, two gold electrodes were deposited over the implanted area, leaving an implanted track in between of size $0.5 \times 5 \mathrm{~mm}^{2}$. The DC surface conductivity measurements were performed with a multimeter and are reported in $\left(\mathrm{k} \Omega \text { square }^{-1}\right)^{-1}$. Since the PDMS is insulating, the surface conductivity directly provides the conductivity of the composite.

While the electrical properties of the gold-implanted samples remain stable with time, the titanium-implanted
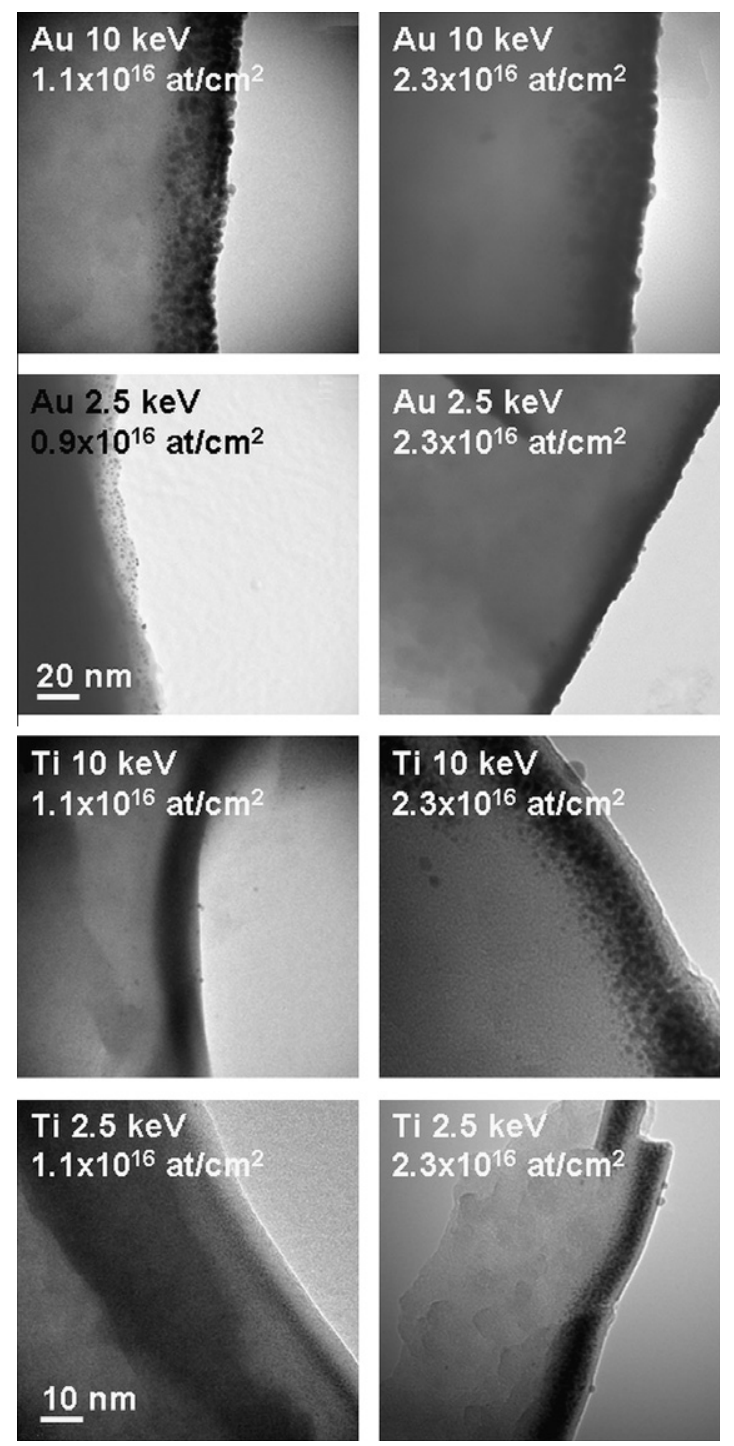

Fig. 3. Cross-section TEM micrographs of FCVA implanted PDMS at 2.5 and $10 \mathrm{keV}$ for $\mathrm{Au}$ and Ti. The doses are $1.1 \times 10^{16} \mathrm{at.}^{-2}$ and $2.3 \times 10^{16}$ at. $\mathrm{cm}^{-2}$. samples oxidize within several hours, depending on the dose. Therefore the results presented here for Ti were taken exactly $15 \mathrm{~min}$ after the implantation.

From TEM images in Figs. 1 and 3, one can observe that the implanted layer for gold ions extends up to the surface of the PDMS, explaining how good electrical contact is readily achieved between the implanted composite and the gold electrodes simply deposited on the PDMS. For titanium samples, the highly implanted layer is situated several nanometers below the surface. Reliable electrical contact with this buried film is nevertheless observed for simple evaporated gold electrodes on the surface of the PDMS, which could be due to conduction through the PDMS region $\sim 5 \mathrm{~nm}$ thick with a lower titanium concentration, but is more likely due to local surface roughness and deformation leading to contact between the top gold electrode and the implanted $\mathrm{Ti}$ layer.

\subsection{Young's modulus measurements}

The samples for the elasticity measurements consist of a $30 \mu \mathrm{m}$ thick layer of PDMS bonded on a $2 \times 2 \mathrm{~cm}^{2}$ Pyrex chip, $500 \mu \mathrm{m}$ thick, with circular through-holes $3 \mathrm{~mm}$ in diameter, which defines the PDMS membranes. After implantation, the chips are mounted on a socket where a pressure of up to $600 \mathrm{~Pa}$ can be applied. The vertical displacement is measured with a white light interferometer (Wyko NT1100, Veeco) as a function of pressure. This method is known as a bulge test [25] and allows the biaxial Young's modulus, residual stress and Poisson's ratio of the membrane to be determined. This setup is optimized for thin elastomer membranes [7].

The membrane consists of the composite (implanted layer) on top of a much thicker non-implanted PDMS membrane. Knowing Young's modulus of PDMS ( $Y_{\text {PDMS }}$ ) and using Eq. (3), one can extract the biaxial Young's modulus of the composite ( $Y_{\text {Comp }}$ ) from that of the membrane $\left(Y_{\text {memb }}\right)$, as follows:

$Y_{\mathrm{Comp}} h_{\mathrm{Comp}}+Y_{\mathrm{PDMS}} \cdot h_{\mathrm{PDMS}}=Y_{\mathrm{memb}} \Rightarrow$

$Y_{\text {Comp }}=\frac{Y-Y_{\text {PDMS }} h_{\mathrm{PDMS}}}{h_{\text {Comp }}}$

where $h_{\text {Comp }}$ and $h_{\text {PDMS }}$ are the normalized thicknesses of the composite and of the underlying non-implanted PDMS layer.

Eq. (4) is only valid if the two layers have the same Poisson ratio, which is not the case for the composite and PDMS. The correct formula is given in Eq. (29) of Ref. [26]. The error one makes by using Eq. (4) and by assuming the same Poisson's ratios for both layers is $<0.5 \%$. Pristine Sylgard 186 PDMS membranes have a Young's modulus of $0.85 \mathrm{MPa}$. 
Table 1

Maximal penetration depth $h_{\text {Comp }}$ as a function of maximum ion energy measured on the gold and titanium-implanted samples.

\begin{tabular}{llll}
\hline Ion energy $(\mathrm{keV})$ & 2.5 & 5 & 10 \\
\hline$h_{\mathrm{Au}}(\mathrm{nm})$ & $18 \pm 1$ & $22 \pm 2$ & $30 \pm 2$ \\
$h_{\mathrm{Ti}}(\mathrm{nm})$ & $13 \pm 1$ & $15 \pm 2$ & $18 \pm 2$ \\
\hline
\end{tabular}

\subsection{Analysis of the composite's structure}

TEM is used to image the microstructure of the composite. Previous work developed a method to prepare crosssections of metal-ion implanted PDMS [27]. Using cryomicrotomy, 60-80 nm thin lamellas (cross-sections) of the gold and titanium-implanted samples were prepared, which were implanted at 2.5, 5 and $10 \mathrm{keV}$. TEM was performed with a Philips CM TEM; see Figs. 1 and 3.

\subsection{Calculation of the metal volume fraction $\phi$}

In what follows, it is assumed that the nanocomposite is uniform throughout its thickness, i.e. that the samples consist of a roughly 50-nm-thick uniform nanocomposite on top of virgin PDMS.

In order to model the physical properties of the nanocomposite with percolation theory, one must know the volume fraction $\phi$ of the implanted metal. This information is obtained from the thickness of the nanocomposite (from TEM images) coupled with the implanted dose. $\phi$ is equal to the ratio $h_{\mathrm{c}} / h_{\text {Comp }}$, where $h_{\mathrm{c}}$ is the thickness of the metal film that would be formed if the ions were all deposited on the surface, and $h_{\text {Comp }}$ is the thickness of the composite. $h_{c}$ is obtained by multiplying the volume of one mole $V_{\text {mole }}$ of ions by the implanted ion dose $D$ :

$h_{c}=V_{\mathrm{mole}} \cdot \frac{D}{N_{\mathrm{Avgd}}}=\frac{A_{r}}{\rho} \cdot \frac{D}{N_{A}}$

where $A_{r}$ is the molar mass ( $196.97 \mathrm{~g} \mathrm{~mol}^{-1}$ for $\left.\mathrm{Au}\right), \rho$ is the density $\left(19.3 \mathrm{~g} \mathrm{~cm}^{-3}\right.$ for $\left.\mathrm{Au}\right)$ and $N_{\mathrm{Avgd}}$ is the Avogadro constant.

The height of the composite $h_{\text {Comp }}$ is taken as equal to the maximal penetration depth of the ions inside the PDMS. In Fig. 1, one can notice that gold ions penetrate the PDMS to a certain depth and form clusters. The maximal penetration depth is defined by a line, after which no clusters can be seen (dashed white line, Fig. 1). This line determines the boundary between the composite and the non-implanted pristine PDMS. For each sample (energy and dose), 10 TEM micrographs were taken, and the maximal penetration depth line was drawn for each one. For each micrograph, the penetration was measured at five places. Since $h_{\text {Comp }}$ does not vary with the dose, the average value was calculated for each energy and element. The results are presented in Table 1 . The incertitude $\Delta h_{\text {Comp }}$ are the variances of the distributions. The maximal error $\Delta \phi$ obtained over all calculated volume fractions is $<3.3 \times 10^{-3}\left(\Delta \phi=h_{c}\right.$ l $\left.h_{\text {Comp }}^{2} \cdot \Delta h_{\text {Comp }}\right)$. The error bars for $Y_{\text {Comp }}$ and for $\sigma_{\text {Comp }}$ are smaller than the dots in the graphs.

\section{Results and discussion}

\subsection{Microstructure of the composite}

Fig. 3 presents TEM images of cross-sections of the gold and titanium-implanted samples. Ions were implanted from the right. On the left is the non-implanted PDMS; the darker region in the center is the implanted layer (composite). The gray region on the right is the carbon TEM grid. For both elements, the ion penetration depth as well as the ion distribution increases with the energy, as expected from TRIM calculations (the Transport of Ions in Matter by J. Ziegler) [28].

The gold ions form rounded gold clusters whose size increases with energy and with dose from 2 to $20 \mathrm{~nm}$ (plotted in Fig. 4b). TEM electron diffraction revealed that the clusters have a crystalline structure (see Fig. 5). The composite formed by gold ion implantations consists of gold clusters in a PDMS matrix.

Titanium, in contrast to gold, does not form metallic clusters, but binds with the PDMS. The nanometer-scale small dark regions seen in the TEM images for Ti at doses $>2.3 \times 10^{16}$ at. $\mathrm{cm}^{-2}$ are amorphous, as determined by
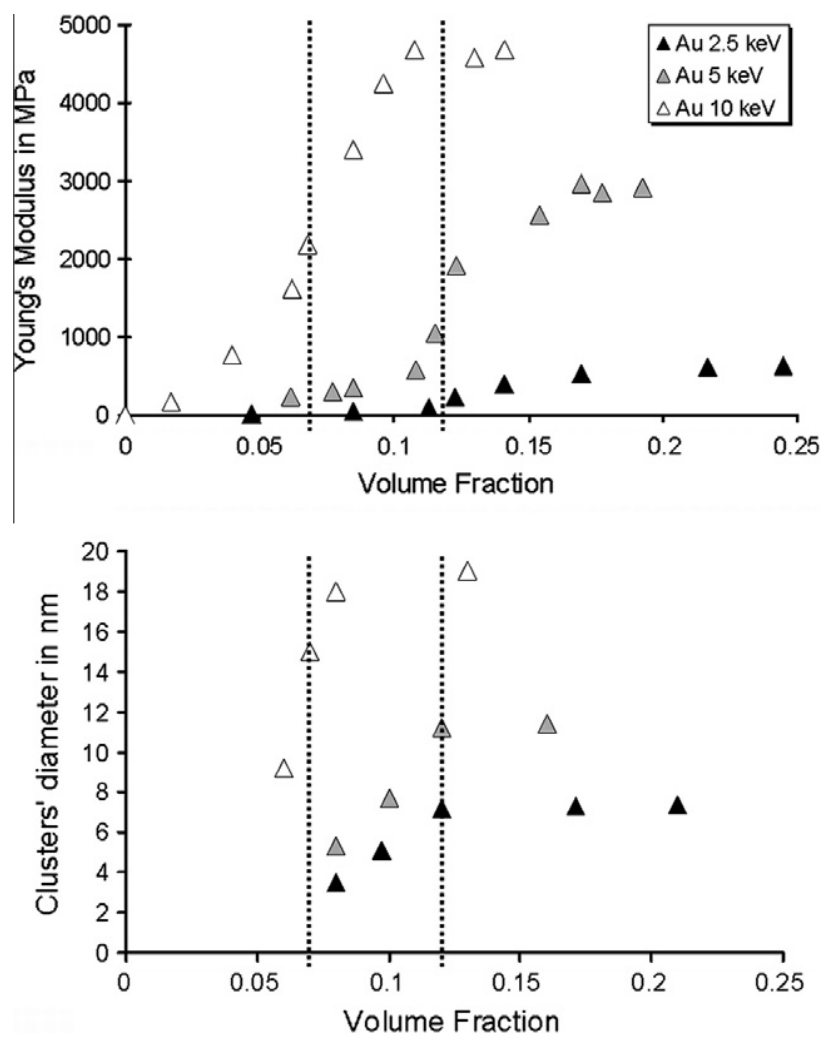

Fig. 4. (a) Young's modulus and (b) cluster diameters for Au-PDMS composite as a function of the metal volume fraction for three ion energies. The volume fraction at which the cluster size saturates corresponds exactly to the percolation threshold for Young's modulus (vertical dashed lines) for all three energies. 

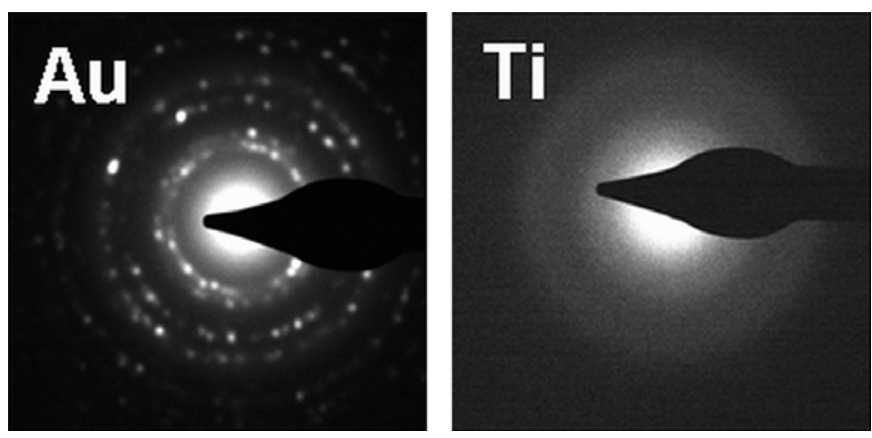

Fig. 5. Electron diffraction patterns for gold and titanium implanted PDMS, showing crystalline structure for gold, and amorphous structure for Ti (TEM parameters: $D=360 \mathrm{~mm}, 200 \mathrm{kV}$, spot 2).

electron diffraction. The size of the dark Ti-rich regions increases with the dose and with energy. At high doses, they form a homogeneous gray layer of highly titanium doped PDMS. The reason for the lack of titanium clusters lies in its electronegativity, which is the lowest (Ti: 1.54) compared with the PDMS constituent atoms ( $\mathrm{Si}: 1.9, \mathrm{H}$ :
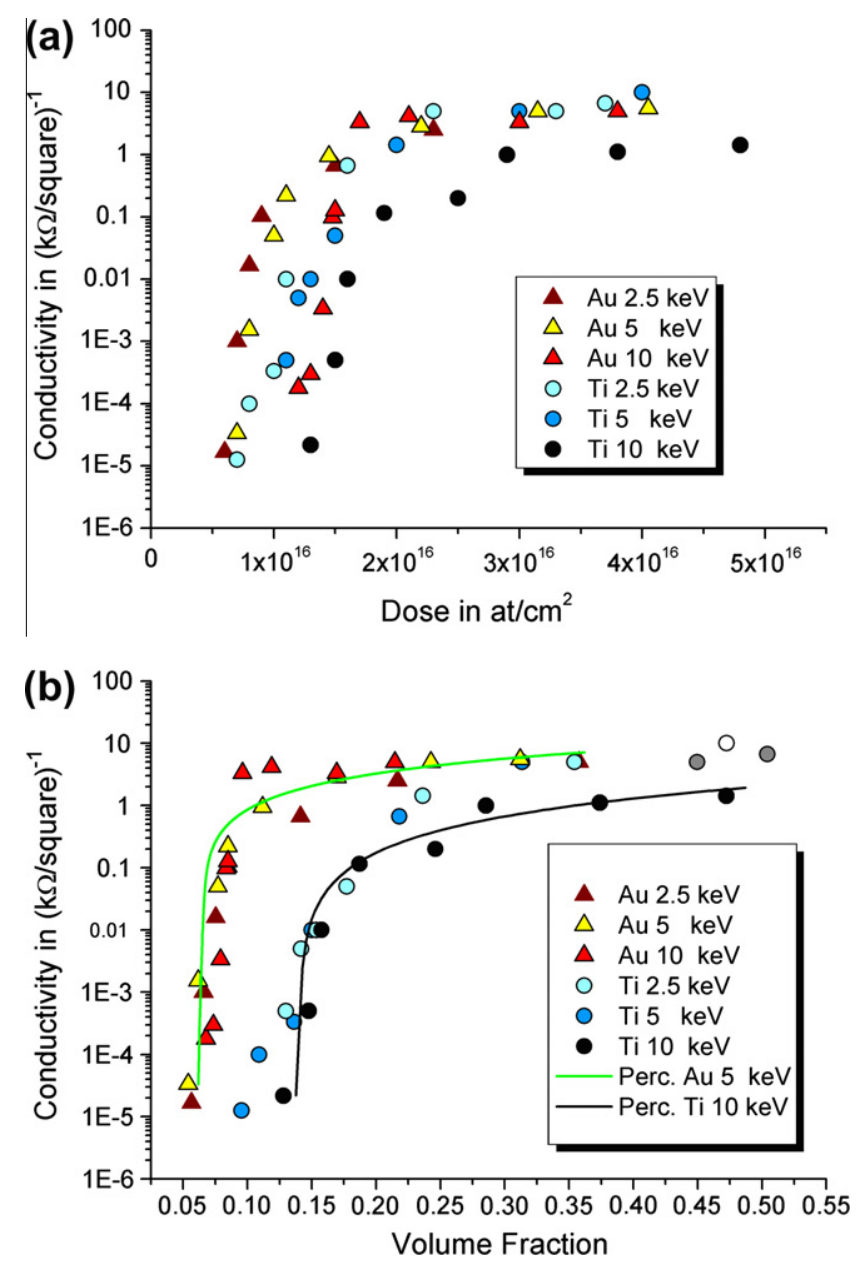

Fig. 6. Electrical conductivity of gold and titanium ions implanted by FCVA at $2.5 \mathrm{keV}, 5 \mathrm{keV}$ and $10 \mathrm{keV}$, as a function of: (a) dose and (b) volume fraction. The two lines in (b) are fits of data with Eq. (1a) for 5$\mathrm{keV}$ gold-implanted samples, and for $10-\mathrm{keV}$ titanium-implanted samples.
2.2, C: 2.55 , O: 3.44) or Au (2.54). Consequently, titanium will bind with the PDMS rather than with itself.

The distribution of the gold clusters is not Gaussian, but shows a higher concentration on and just beneath the surface than deep below it. The main reason for this lies in the operation of the FCVA, in which the ion energy drops during each pulse from the initial well-defined value given by the accelerating potential. Consequently, only the first ions per pulse have the predetermined energy.

\subsection{Electrical conductivity}

\subsubsection{Results}

The electrical conductivity $\sigma_{\text {Comp }}$ of the nanocomposite samples is presented in Fig. 6. In Fig. 6a, the horizontal axis is the dose. The behavior shows the general shape expected for percolative systems with a steep rise in the electrical conductivity $\sigma$ at the percolation threshold. The doses at which this occurs range from $0.6 \times 10^{16}$ to $1.3 \times 10^{16}$ at. $\mathrm{cm}^{-2}$ and, for the same element increases with ion energy. The lowest measurable value for $\sigma$ was $1.3 \times 10^{-5}\left(\mathrm{k} \Omega \text { square }^{-1}\right)^{-1}$. At higher doses, the conductivity saturates near $1\left(\mathrm{k} \Omega \text { square }^{-1}\right)^{-1}$.

In Fig. 6b, the same conductivity is plotted vs. volume fraction $\phi$, which is the relevant variable for percolation theory. Below the percolation threshold, the conductivity could not be measured with the standard multimeter, and it was assumed that $\sigma_{\text {PDMS }}$ (or $\sigma_{i}$ in Eq. (1b)) is zero. The two solid lines in the figure are fits to the percolation model in Eq. (1a). The fitting parameters are given in Table 2. A clear difference between gold and titanium samples can be seen and, for each metal, the data for the three energies now lie on top of each other. The average gold particle size is 7, 11 and $19 \mathrm{~nm}$ at the percolation threshold for ion energies of $2.5,5$ and $10 \mathrm{keV}$ (Fig. $4 \mathrm{~b}$ ), yet the $\sigma_{\text {Comp }}$ vs. $\phi$ curves have very similar percolation parameters for the three energies.

\subsubsection{Discussion}

4.2.2.1. Fitted resistivity. Transforming the fitted $\sigma_{c}$ into the volume resistivity $\rho$ using the thickness of the nanocomposite, one obtains $\rho_{\mathrm{Au}}^{\text {percolation }}=300 \mathrm{n} \Omega \mathrm{m} \quad$ and $\rho_{\mathrm{Ti}}^{\text {percolation }}=812 \mathrm{n} \Omega \mathrm{m}$ In the simplest scenario, the conductivity values obtained from the percolation fits would be

Table 2

Fitted percolation parameters for conductivity for gold and titanium implanted PDMS: percolation threshold $\phi_{c}$, exponent $t$ and conductivity $\sigma_{c}$ expressed in $\left(\mathrm{k} \Omega \text { square }^{-1}\right)^{-1}$ for three ion energies.

\begin{tabular}{llccc}
\hline & & $2.5 \mathrm{keV}$ & $5 \mathrm{keV}$ & $10 \mathrm{keV}$ \\
\hline $\mathrm{Au}$ & $\phi_{c}$ & $0.071 \pm 0.004$ & $0.064 \pm 0.006$ & $0.085 \pm 0.004$ \\
& $\sigma_{c}$ & $22 \pm 5$ & $24 \pm 5$ & $25 \pm 4$ \\
& $t$ & $1.2 \pm 0.1$ & $1 \pm 0.1$ & $0.8 \pm 0.1$ \\
$\mathrm{Ti}$ & $\phi_{c}$ & $0.12 \pm 0.03$ & $0.11 \pm 0.02$ & $0.13 \pm 0.03$ \\
& $\sigma_{c}$ & $18 \pm 9$ & $19 \pm 8$ & $8 \pm 8$ \\
& $t$ & $1.1 \pm 0.4$ & $1.6 \pm 0.4$ & $1.4 \pm 0.4$ \\
\hline
\end{tabular}


equal to the bulk resistivity values, namely $22 \mathrm{n} \Omega \mathrm{m}$ for gold and $420 \mathrm{n} \Omega \mathrm{m}$ for titanium. The lower conductivity determined for the implanted metals indicates that the conduction mechanism is strongly influenced by the microstructure, i.e. by resistance between nanoparticles, as well as the conductivity of such small particles, which can be much less than bulk. It has been shown, for instance, that $<3 \mathrm{~nm}$ gold particles are insulating and that only $>10 \mathrm{~nm}$ gold approaches bulk electrical properties [30].

Owing to the structure of the composites and weak temperature dependence, the conduction mechanism for goldPDMS composite can be interpreted as Ohmic conduction between gold clusters. The titanium implantation can be interpreted as a doping of the elastomer rather than including metal nanoparticles. The Ti-doped PDMS becomes conducting as the Fermi levels are shifted from the corresponding bandgaps towards the conduction bands. Further detailed analysis of the voltage, frequency, magnetic field and temperature dependence of current would be needed to distinguish the resistivity of nanometer-size particles clearly from the inter-particle resistance.

4.2.2.2. Percolation threshold. For gold and titanium samples, the percolation thresholds $\phi_{c}$ are seen to be independent of the ion energy (within the fitting error). The percolation threshold occurs at a volume fraction between 0.064 and 0.085 for gold, and between 0.11 and 0.13 for titanium. The observed maximal $\phi_{c}$ values for gold and titanium samples are $<0.16$ (predicted for the model system of touching impenetrable spheres [31]), but is well in the range of what is reported for carbon-powder-rubber-based composites) [32,33].

Balberg showed that, if the particles are not perfectly spherical, $\phi_{c}$ is usually $<0.16$ [34]. Others have found that, if the particles are interacting (i.e. sticky spheres), the effect of attractive forces on impermeable objects is to lower $\phi_{c}$ [35]. Non-isotropic composites have non-universal values of $\phi_{c}$ [19], which is relevant for the gold samples, in which the biggest clusters are found on the surface, and only deep inside PDMS can one observe small clusters. This phenomenon is less pronounced for titanium samples, which have higher $\phi_{c}$.

Another explanation for the $\phi_{c}$ value is that metal particles at nanometer-scale exhibit different physical properties from those of bulk [36]. If the metal cluster conductivity changes with volume fraction, $\phi_{c}$ will be affected both in an absolute manner, as well as simply from a fitting perspective.

4.2.2.3. Exponent. The exponent $t$ ranges for the titanium samples from 1.1 to 1.6 , and for the gold implantations from 0.8 to 1.2 , with no clear energy dependence; see Table 2. In $2 \mathrm{D}$, the theoretical value of $t$ is 1.3 , which is closer to the measurements than the 3D value of 2. Indeed, comparing the thicknesses of the gold composite (e.g. $18 \mathrm{~nm}$ for $2.5 \mathrm{keV})$ with the clusters' size $(7 \mathrm{~nm}$ for $2.5 \mathrm{keV}$ ), it is very likely that the conductivity is more $2 \mathrm{D}$ than 3D. However, owing to the structure of titanium samples (well distributed and mixed with PDMS without creation of clusters), one expects these composites to exhibit 3D conductivity and thus have a higher exponent that is closer to $3 \mathrm{D}$ theoretical value.

Experiments have shown that particles with diverse shapes and anisotropic conductivities can lead to different and often anisotropic exponents for transverse and inplane measurements of conductivity [37]. The higher the anisotropy, the further away the exponent from the universal value. As presented in Fig. 3, titanium ions are well incorporated in the molecular structure of PDMS and form a homogeneous composite without creating clusters. They are well distributed under the surface. The exponents are closer to 2. Gold clusters vary in size with depth - they are bigger next to the surface. Their shape is not perfectly spherical, but rather ellipsoidal, elongated parallel to the surface and, according to Deprez, lower values of $t$ are expected for such a case [37]. Celzard and Marêché showed that isotropy of conductivity, particularly in the percolation domain, is required to obtain universal values, namely 2 in 3D [19]. Since these effects (anisotropy and poly-dispersion) increase with energy, this could explain the slight decrease in the exponent with the energy.
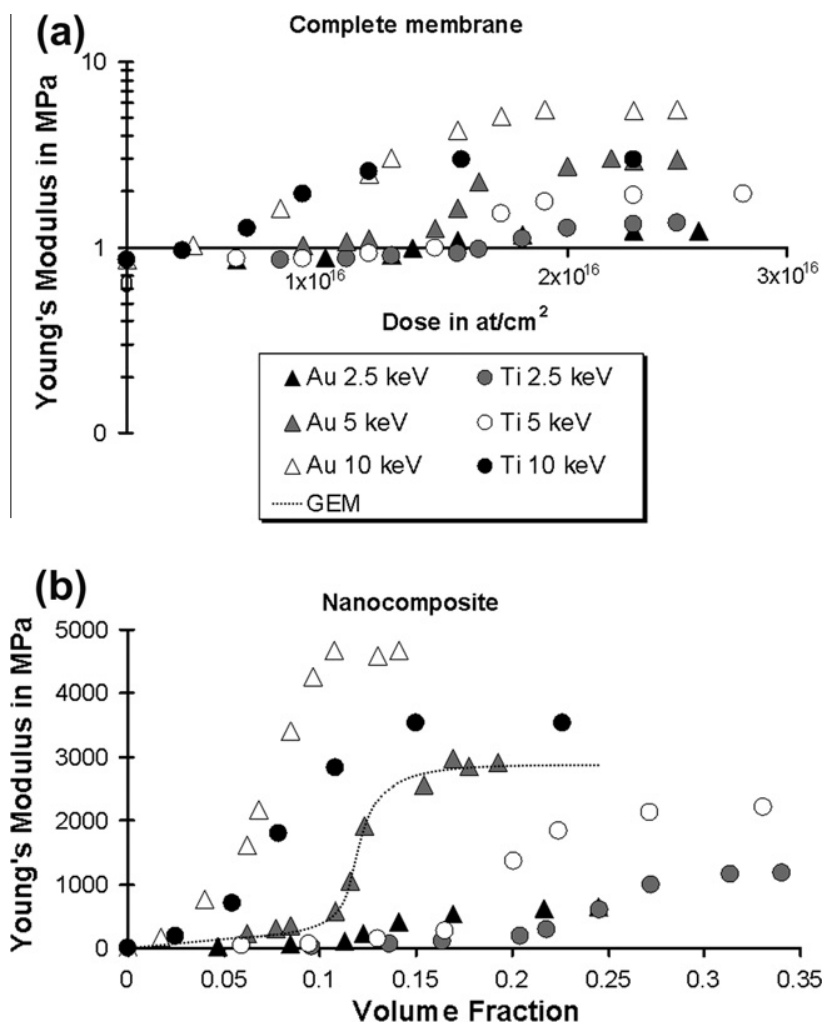

Fig. 7. Young's modulus of gold and titanium samples implanted at $2.5 \mathrm{keV}, 5 \mathrm{keV}$ and $10 \mathrm{keV}$ : (a) raw bulge test measurements of the complete $30-\mu \mathrm{m}$-thick membrane as a function of the dose; (b) Young's modulus only of the composite, calculated with laminar theory (Eq. (4)) as a function of the metal volume fraction. The curve is the fit of data with the GEM (Eq. (2)) for $5 \mathrm{keV}$ gold-implanted samples. 
To conclude, on the percolation parameters for conductivity, the measured $\phi_{c}$ and $t$ are lower than the theoretical values, but are in line with what is reported for other nanosized composites.

\subsection{Young's modulus $Y$}

\subsubsection{Results}

Unlike the conductivity data, Young's modulus data can be fitted both above and below the percolation threshold. Fig. $7 \mathrm{a}$ presents $Y_{\text {memb }}$ of the implanted membrane obtained with the bulge test setup as a function of the ion dose. The laminar theory (Eq. (3)) was then applied to determine Young's modulus $Y_{\text {Comp }}$ of only the $\sim 50$ nm-thick composite.

Fig. 7b shows the extracted $Y_{\text {Comp }}$ of the nanocomposite as a function of the volume fraction, displaying classical percolation behavior. This allows percolation theory (Eqs. (1a) and (1b)) to be used to determine $\phi_{c}$, the two exponents ( $f$ for $\phi>\phi_{c}, a$ for $\phi<\phi_{c}$ ), as well as Young's modulus of the components of the composite: $Y_{\mathrm{Au}}^{\text {perc }}, Y_{\mathrm{Ti}}^{\text {perc }}$ and $Y_{\text {PDMS }}^{\text {perc }}$. The results are summarized in Table 3. The experimental results presented in Fig. $7 \mathrm{~b}$ have also been fitted with the GEM (Eq. (2)), yielding the same fitting parameters as for percolation theory.

Fig. 7a shows that $Y_{\text {memb }}$ of the full PDMS membrane increases with the ion dose and energy, saturating at the doses $>2 \times 10^{16}$ at. $\mathrm{cm}^{-2}$. The Young's moduli of all the membranes are $<6 \mathrm{MPa}$. The highest and the lowest saturation values are obtained for the membranes implanted with gold ions: $5.5 \mathrm{MPa}$ at $10 \mathrm{keV}$ and $1.2 \mathrm{MPa}$ at $2.5 \mathrm{keV}$, respectively.

The samples implanted at the highest energy exhibit modifications in properties that scale linearly with dose or volume fraction.

\subsubsection{Discussion}

The Young's modulus depends directly on the physicalmechanical influence caused by the implanted metal particles and on the radiation-induced modifications of the polymer structure. The latter are not taken account by the percolation theory. Radiation damage of the PDMS did not impact the conductivity data, as the PDMS remained insulating, but plays an important role in the mechanical properties of the composite. When an energetic ion impacts the PDMS, it induces changes in the chemistry of the polymer by chain-scission and cross-linking [29], leading to increased density and reduced molecular weight, and to higher stiffness.

The metals ( $\mathrm{Ti}$ and $\mathrm{Au}$ ) have Young's modulus five orders of magnitude greater than PDMS.

4.3.2.1. Percolation threshold. The percolation threshold was obtained twice for each ion type (at high and low volume fraction, e.g. Eqs. (1a) and (1b), and the two values of $\phi_{c}$ agree within the fitting error, a good indication that the same theory applies over the entire experimentally accessed volume fraction range.

The percolation threshold for the titanium-implanted samples decreases from 0.25 at $2.5 \mathrm{keV}$ to 0.1 at $10 \mathrm{keV}$, and for the gold composites it decreases from 0.13 at $2.5 \mathrm{keV}$ to 0.06 at $10 \mathrm{keV}$. All the percolation thresholds correspond approximately to doses of $1.5 \times 10^{16}$ at. $\mathrm{cm}^{-2}$, except for gold and titanium samples implanted at $10 \mathrm{keV}$.

In Fig. $4 \mathrm{a}, Y_{\text {Comp }}$ of gold samples is plotted vs. volume fraction $\phi$, and in Fig. $4 \mathrm{~b}$ the average size of the gold clusters is plotted vs. $\phi$ for three different ion energies. The gold cluster size increases with $\phi$, then saturates. $\phi_{c}$ (vertical lines) is exactly in the domain where the clusters' diameter saturates, for all three energies, showing a direct link between cluster size evolution and $\phi_{c}$. One simple explanation is that the clusters cease to grow once they touch, which would also correspond to a rapid increase in $Y_{\text {Comp }}$. It is observed that the larger clusters reach percolation at a lower $\phi$ than smaller clusters.

It is interesting to note that, at a given $\phi$, the ratio between the cluster sizes at different ion energies $(E)$ is the same as the ratio of the energies. This can be attributed to the cluster diameter depending on the ion mobility, which is directly related to $E$.

Table 3

Fitted percolation parameters and Young's modulus as a function of energy for gold and titanium implanted PDMS: percolation threshold $\phi_{c}$, Young's moduli of the constituents (in MPa) and exponents $a$ and $f$.

\begin{tabular}{|c|c|c|c|c|c|}
\hline & & $2.5 \mathrm{keV}$ & $5 \mathrm{keV}$ & $10 \mathrm{keV}$ & Fitting error \\
\hline $\begin{array}{l}\mathrm{Au} \\
\left(\phi<\phi_{c}\right)\end{array}$ & $\begin{array}{l}\phi_{c} \\
a \\
Y_{\mathrm{PDMS}}^{\text {perc }}\end{array}$ & $\begin{array}{l}0.13 \\
0.9 \\
2\end{array}$ & $\begin{array}{l}0.13 \\
0.9 \\
3\end{array}$ & $\begin{array}{l}0.07 \\
1.2 \\
2\end{array}$ & $\begin{array}{l} \pm 0.01 \\
\pm 0.3 \\
\pm 1\end{array}$ \\
\hline $\begin{array}{l}\mathrm{Au} \\
\left(\phi>\phi_{c}\right)\end{array}$ & $\begin{array}{l}\phi_{c} \\
f \\
Y_{\mathrm{Au}}^{\text {perc }}\end{array}$ & $\begin{array}{l}0.12 \\
0.2 \\
1080 \pm 270\end{array}$ & $\begin{array}{l}0.12 \\
0.2 \\
4850 \pm 1210\end{array}$ & $\begin{array}{l}0.05 \\
0.7 \\
24,700 \pm 6180\end{array}$ & $\begin{array}{l} \pm 0.01 \\
\pm 0.1\end{array}$ \\
\hline $\begin{array}{l}\mathrm{Ti} \\
\left(\phi>\phi_{c}\right)\end{array}$ & $\begin{array}{l}\phi_{c} \\
f \\
Y_{\mathrm{Ti}}^{\text {perc }} \\
\end{array}$ & $\begin{array}{l}0.24 \\
0.2 \\
1700 \pm 430\end{array}$ & $\begin{array}{l}0.20 \\
0.1 \\
2810 \pm 700\end{array}$ & $\begin{array}{l}0.08 \\
0.1 \\
4740 \pm 1190\end{array}$ & $\begin{array}{l} \pm 0.02 \\
\pm 0.1\end{array}$ \\
\hline
\end{tabular}


Titanium ions do not form clusters, but reinforce PDMS on a molecular level, leading to a homogeneous composite. The bigger the energy, the bigger the distribution of the ions and the thicker the composite layer.

4.3.2.2. Exponent. The theoretical value for exponent $a$ is 0.65 . The experimental value obtained for gold samples is relatively close to this value $(a \approx 1)$, where for the titanium implantations it reaches values between 1.3 and 2.3. As shown in Table 3, there is no strong energy dependence of the $a$ exponent.

The higher $a$ exponent for $\mathrm{Ti}$ samples means a lower slope when approaching $\phi_{c}$ from the lower volume fractions. Gold ions transfer larger collision energies to PDMS than titanium ions do, and therefore induce bigger chemical changes in the molecular structure of PDMS. These energy effects are added to the direct mechanical effects caused by the volume of particles. This can explain the steeper percolation curve for gold implantations.

The value of the exponent $f\left(\phi>\phi_{c}\right)$ lies between 0.1 and 0.3 for both elements. The exception is $10 \mathrm{keV}$ implanted titanium, with $f \approx 0.7$. These values are much lower than the numerical simulations $(\sim 3.75$ obtained for the general continuum percolation model) [12]. Two main arguments are presented to explain this apparent discrepancy. Both arguments relate to the data taken at $\phi$ well above $\phi_{c}$. The first explains the measured results; the second explains the fitting results.

First, consider the radiation-induced chemical changes in the polymer. These scale roughly linearly with ion dose, becoming important particularly above $\phi_{c}$. This has the main effects of an increase in Young's modulus of the irradiated PDMS, leading to a steeper percolation slope with lower exponents. This dynamic modification of the composite components is not considered in percolation theory. Consequently, the exponents $a$ that are related to the percolation curve below $\phi_{c}$ are in the neighborhood of the universal value, and the exponents $f$ that are related to the percolation curve above $\phi_{c}$ are further from their universal value.

The second argument is made by introducing the notion of the crossover region [16]. If the ratio of the properties of the two components is not zero, e.g. if $Y_{i} / Y_{c} \neq 0$ (where $i=$ insulator and $c=$ conductor), the properties must be considered on a more equal footing by limiting the applicability of the theory to a volume fraction range near $\phi_{c}$. The width of this region $\delta=\left|\phi-\phi_{c}\right|$ is given by $\delta=\left(Y_{i} / Y_{c}\right)^{1 /(a+f)}$. From the tabled Young's modulus of PDMS $\left(Y_{i}=0.85 \mathrm{MPa}\right)$ and metals $\left(Y_{c} \approx 100 \mathrm{GPa}\right.$ ), using universal values for $a$ and $f$, one obtains $\delta \approx 0.08$. Applying this crossover region approach, and limiting the fitting of Eqs. (1a) and (1b) to data points near $\phi_{c}$ rather than considering all the data, the fitted $f$ exponent increases. For $2.5 \mathrm{keV}, 5 \mathrm{keV}$ and $10 \mathrm{keV}$, the newly fitted values for $f$ are $3 \pm 0.3(\delta=0.06), 2.8 \pm 0.4(\delta=0.04)$ and $2.7 \pm 0.3(\delta=0.06)$, much closer to the universal value. From Fig. $6 \mathrm{~b}$, one can notice that this procedure will not change the $a$ values significantly, because below $\phi_{c}$ the results are continuously increasing with the dose, without saturation.

4.3.2.3. Fitted Young's modulus of components. Percolation theory was used to determine Young's modulus of the components of the composite from the percolation fits, i.e. $Y_{\mathrm{Au}}^{\text {perc }}, Y_{\mathrm{Ti}}^{\text {perc }}$ and $Y_{\mathrm{PDMS}}^{\text {perc }}$.

For gold clusters, $Y_{\mathrm{Au}}^{\mathrm{perc}}$ increases with cluster size (itself related to energy, see discussion above), starting at $1 \mathrm{GPa}$ for a 7-nm cluster diameter and reaching $25 \mathrm{GPa}$ for a 19-nm cluster diameter (Table 3). Compared with bulk $\mathrm{Au}(80 \mathrm{GPa})$, the highest fitted values of the metal's Young modulus are roughly 25 times smaller. But the bulk values are not valid for nanometer-size scale: Schaefer et al. obtained Young's modulus of the same range $(10 \mathrm{GPa}$ for $10 \mathrm{~nm}$ ) for nanometer-size gold clusters that were preformed in the gas phase and deposited on atomically flat substrates [38]. The strong increase in $Y_{\mathrm{Au}}^{\text {perc }}$ with energy (hence with particles' size) is thus mainly linked to the strong size dependence of the mechanical properties of nanometer-scale particles.

$Y_{\mathrm{Ti}}^{\text {perc }}$ increases with energy from $1.7 \mathrm{GPa}$ at $2.5 \mathrm{keV}$ to $4.7 \mathrm{GPa}$ at $10 \mathrm{keV}$, values much lower than for bulk titanium $(116 \mathrm{GPa})$. The titanium samples are doped PDMS rather than microparticle-reinforced PDMS, as was the case for the gold-implanted samples. The increase in $Y_{\mathrm{Ti}}^{\text {perc }}$ with energy can be explained by the associated increase in the size of the titanium aggregates, and by the indirect effects of the chemical changes in PDMS.

Starting with the PDMS initial value of $0.85 \mathrm{MPa}$, $Y_{\mathrm{PDMS}}^{\text {perc }}$ increased after implantation to $2 \mathrm{MPa}$, which is a very reasonable value, given the effects of radiation on the polymer structure. The value obtained also gives additional confidence in the values for $Y_{\mathrm{Au}}^{\text {perc }}$ and $Y_{\mathrm{Ti}}^{\text {perc }}$ since, to obtain these values, first the laminar theory was used to extract $Y_{\text {Comp }}$ from $Y_{\text {memb }}$, and then the percolation theory was used to determine Young's modulus of the components of the composite.

\subsection{Comparison between Young's modulus and conductivity}

Metal-ion implanted PDMS is one of the rare physical systems where percolation theory can be applied to model two physical properties at once: Young's modulus $Y$ and electrical conductivity $\sigma$. These two properties are both related to the structure of the composite reflecting the percolation behavior. In the simplest model, the percolation thresholds should be the same for $\sigma$ and for $Y$.

Both $Y$ and $\sigma$ are influenced by two implantationinduced effects. The first is the addition of metal atoms in the elastomer matrix (i.e. the increase in metal volume fraction as the dose increases), and the second is the radiationinduced chemical modifications of the molecular structure of the PDMS (chemical changes).

While the percolation threshold $\phi_{c}^{\text {Young }}$ for Young's modulus decreases with ion energy and there is no clear distinction between $\mathrm{Au}$ and $\mathrm{Ti}$ samples, the percolation 
threshold $\phi_{c}^{\text {conduct }}$ for electrical conductivity is clearly distinct for the titanium and gold samples and is independent of ion energy. The reason for this different behavior for $\phi_{c}^{\text {Young }}$ and $\phi_{c}^{\text {conduct }}$ is due to the influences on $Y$ and $\sigma$ of implantation-related physico-mechanical changes (e.g. particle's volume and connectivity), chemical changes (e.g. density), composite structure (e.g. clusters), as well as the intrinsic element properties (e.g. bulk conductivity and oxidation). For instance, $Y$ is directly related to the material structure, e.g. particle formation in a form of clusters $(\mathrm{Au})$ or amorphous aggregates $(\mathrm{Ti})$ which depends on the ion energy. $\sigma$ depends on the connectivity of the particles and on the conduction mechanism, which do not directly depend on the energy. The influence of the implanted particles on the mechanical properties already starts in a continuous manner with the first implanted ion. However, the electrical properties of the composite change only after a certain ion concentration has been exceeded in the case of titanium doping, or after a certain particle size has been reached in the case of the gold clusters.

For the exponents, different arguments are used to explain their generally low values, and it is important to note here that these explanations are coherent for the electrical and mechanical properties. For conductivity, three arguments are used to explain the exponents: the poly-dispersion of the particles, the anisotropy of the composite and the dimensionality of the particle connectivity. The first two also explain the low exponents of $Y$. The third does not influence Young's modulus explanations that are based on the volume effects of the particles and not on their connectivity.

For the exponents determined for $Y$, in addition to the first two explanations above, their values can be explained by the crossover region and the radiation-induced chemical changes in the polymer structure. The radiation-induced stiffening only has no significant impact on conductivity. The argument based on the crossover region modifies only $Y$ values above the percolation threshold. The values below the threshold stay almost unchanged. Applying the "crossover region" term to the electrical measurements would leave the results for the gold samples unchanged, but it would also increase the conductivity exponent for titanium composites, and it would approach the 3D value of 2 . Thus, for both physical properties the argument of the crossover region can be used and yields values proximal to the universal ones.

\section{Conclusions}

The mechanical and electrical properties of a $50-\mathrm{nm}$ thick metal-elastomer nanocomposite formed by metalion implantation of PDMS were presented for $\mathrm{Au}$ and $\mathrm{Ti}$ ion doses from $0.1 \times 10^{16}$ at. $\mathrm{cm}^{-2}$ to $5 \times 10^{16}$ at. $\mathrm{cm}^{-2}$, and the energies of $2.5 \mathrm{keV}, 5 \mathrm{keV}$ and $10 \mathrm{keV}$. Using TEM micrographs, the metal volume fraction of the composite could be determined, allowing the conductivity and Young's modulus to be plotted vs. metal volume fraction.
This permitted, for the first time, quantitative use of the percolation theory for nanocomposites based on ion implantation. The percolation fits were discussed with the structural analysis obtained by TEM and are in line with what is reported for other nanosized composites. Percolation thresholds and exponents for both electrical and mechanical properties were determined on the same nanocomposite samples, and were found to differ significantly for the different properties. While both the conductivity and the stiffness of these composites are well explained by percolation theory, the interactions between metal nanoparticles have different effects in determining the mechanical and electrical properties of the composite.

\section{Acknowledgment}

Financial support from the Swiss National Science Foundation Grants 200020-120164 and 200020-130453 is acknowledged.

\section{References}

[1] Niu XZ, Peng S, Wen W, et al. Adv Mater 2007;19:2682.

[2] Rwei SP, Ku FH, Cheng KC. Colloid Polym Sci 2002;280:1110.

[3] Khosla A, Gray B. Mater Lett 2009;63:1203.

[4] Urdaneta MG, Delille R, Smela E. Adv Mater 2007;19:2629.

[5] Salvadori MC, Cattani C, Teixeira FS, Brown IG. Appl Phys Lett 2008;93:073102.

[6] Teixeira FS, Salvadori MC, Cattani M, Brown IG. J Appl Phys 2009; 106:056106.

[7] Rosset S, Niklaus M, Dubois P, et al. Adv Funct Mater 2008;19:470.

[8] Rosset S, Niklaus M, Dubois P, et al. J Microelectromech Syst 2009; 18:1300.

[9] Watt JP, Davies GF, O'Connell RJ. Revs Geophys Space Phys 1976;14:541.

[10] Torquato S. Int J Solids Struct 2000;37:411.

[11] Maxwell JC. Treatise on electricity and magnetism. Oxford: Clarendon Press; 1873.

[12] Torquato S. Random heterogeneous materials, microstructure and macroscopic properties. New York: Springer; 2002.

[13] Broadbent S, Hammersley J. Proc Camb Phil Soc 1957;53:629.

[14] Benguigui L, Ron P. Phys Rev Lett 1993;70:2423.

[15] Sahimi M. Applications of percolation theory. London: Taylor; 1994.

[16] Stauffer S, Aharony A. Introduction to percolation theory. London: Taylor; 1994.

[17] Ce-Wen N. Prog Mater Sci 1993;37:1.

[18] Golden K. Phys Rev Lett 1990;65:2923.

[19] Celzard A, Marêché JF. Physica A 2003;317:305.

[20] McLachlan DS, Blaszkiewicz M, Newnham RE. J Am Ceram Soc 1990;73:2178.

[21] Ward IM, Sweeney J. An introduction to the mechanical properties of solid polymers. London: John Wiley; 2004.

[22] www.rhk-tech.com.

[23] Anders A, Yushkov GY. J Appl Phys 2002;91:4824.

[24] Brown IG, Monteiro OR, Bilek MMM. Appl Phys Lett 1999;74:2426.

[25] Vlassak JJ, Nix WD. J Mater Res 1992;7:3242.

[26] Liu B, Feng X, Zhang SM. Compos Sci Technol 2009;69:2198.

[27] Niklaus M, Rosset S, Dubois P, et al. Scripta Mater 2008;59:893.

[28] Niklaus M, Rosset S, Dubois P, et al. In: Mater res soc symp proc, vol. 1188; 2009. p. LL03.

[29] Fink D. Fundamentals of ion-irradiated polymers. Berlin: Springer; 2004.

[30] Johnston RL. Atomic and molecular clusters. London: Taylor; 2002.

[31] Sher H, Zallen R. J Chem Phys 1970;53:3759. 
[32] Kofod G, McCarthy DN, Stoyanov H. Appl Phys Lett 2009;94:232905.

[33] Dang ZM, Xial B, Yao SH. Appl Phys Lett 2009;94:042902.

[34] Balberg I. Phys Rev Lett 1987;59:1305.
[35] Seaton NA, Glandt ED. J Chem Phys 2006;86:4668.

[36] Marquardt P, Nimtz G. Phys Rev B 1991;43:14245.

[37] Deprez N. J Phys 1991;70:3681.

[38] Schaefer DM, Patil A, Andres RP, et al. Phys Rev B 1995. 